\title{
Novel and recurrent ALDH3A2 mutations in Italian patients with Sjögren-Larsson syndrome
}

\author{
Biagio Didona - Andrea Codispoti - Enrico Bertini - Wiliam B. Rizzo • \\ Gael Carney · Giovanna Zambruno - Carlo Dionisi-Vici - Mauro Paradisi · \\ Cristina Pedicelli · Gerry Melino $\cdot$ Alessandro Terrinoni
}

Received: 4 May 2007/Accepted: 13 July 2007/Published online: 28 September 2007

(C) The Japan Society of Human Genetics and Springer 2007

\begin{abstract}
Sjögren-Larsson syndrome (SLS; MIM\#270200) is an autosomal recessive neurocutaneous disease caused by mutations in the $A L D H 3 A 2$ gene for fatty aldehyde dehydrogenase (FALDH), a microsomal enzyme that catalyzes the oxidation of medium- and long-chain aliphatic aldehydes to fatty acids. We studied two unrelated Italian SLS patients with ichthyosis, developmental delay, spastic diplegia and brain white matter disease. One patient was homozygous for a novel $A L D H 3 A 2$ insertion mutation (c.767insA) in exon 5. The other SLS patient was a compound heterozygote for two previously reported mutations: a splice-site mutation $(c .471+2 \mathrm{~T}>\mathrm{G})$ in intron 3 and a missense mutation (c.1094C $>\mathrm{T}$; S365L) in exon 7 . Analysis of fibroblast RNA by RT-PCR indicated that the splice-site mutation caused skipping of exons 2 and 3. The
\end{abstract}

Biagio Didona and Andrea Codispoti equally contributed to the work.

B. Didona

I Dermatology Division, IDI-IRCCS,

Via Monti di Creta, 104, Rome, Italy

A. Codispoti · G. Melino $(\bowtie) \cdot$ A. Terrinoni $(\bowtie)$

Department of Experimental Medicine, IDI-IRCCS

Biochemistry Laboratory, c/o University of Tor Vergata,

Via Montpellier 1, 00133 Rome, Italy

e-mail: gerry.melino@uniroma2.it

A. Terrinoni

e-mail: alessandro.terrinoni@uniroma2.it

E. Bertini · C. Dionisi-Vici

Department of Laboratory Medicine,

Unit of Molecular Medicine,

Bambino Gesu Hospital, Rome, Italy

W. B. Rizzo - G. Carney

University of Nebraska Medical Center, Omaha, NE, USA
c.1094C > T mutation, previously associated with two $A L D H 3 A 2$ haplotypes, was found on a third distinct haplotype in our patient, which indicates that it arose independently in this kindred. These results add to understanding of the genetic basis of SLS and will be useful for DNA diagnosis of this disease.

Keywords Ichthyosis - Mental retardation . Spastic diplegia - Mutation - Leukotriene . $\omega$-Oxidation $\cdot$ Fatty aldehyde $\cdot$ Fatty alcohol

\section{Introduction}

Sjögren-Larsson syndrome (SLS; MIM\#270200) is a rare autosomal recessive disorder characterized by mental retardation, spastic di- or tetraplegia and congenital ich-

G. Zambruno

Molecular Biology Laboratory, IDI-IRCCS,

Via Monti di Creta, 104, Rome, Italy

M. Paradisi · C. Pedicelli

VII Dermatology Division, IDI-IRCCS,

Via Monti di Creta, 104, Rome, Italy

G. Melino

Medical Research Council, Toxicology Unit,

Leicester University, Hodgkin Building,

Lancaster Road, P.O. Box 138, Leicester LE1 9HN, UK

G. Melino

Mondino-Tor Vergata Center for Experimental Neurobiology,

University of Rome Tor Vergata, Rome, Italy 
thyosis (Jagell et al. 1981; Rizzo 2001; Sjogren and Larsson 1957). The syndrome has a high prevalence in northern Sweden where it was first described, but it is also found worldwide. Mental retardation has variable severity and is profound in at least one-third of cases (Rizzo 2001). The ichthyosis in SLS is usually present at birth, although it can appear later in the 1st year of life (Jagell et al. 1981). Retinal glistening white dots and pigmentary degeneration are often present, and photophobia is common (Jagell et al. 1980).

SLS is caused by mutations in the $A L D H 3 A 2$ gene that codes for fatty aldehyde dehydrogenase (FALDH) (De Laurenzi et al. 1996). The $A L D H 3 A 2$ gene is located on chromosome 17p11.2 (Pigg et al. 1994; Rogers et al. 1995) and consists of 11 exons encoding a protein of 485 amino acids (Chang and Yoshida 1997). Alternative splicing of the gene generates a second minor transcript that codes for a variant FALDH protein of 508 amino acids possessing a unique carboxy-terminus (Rogers et al. 1997). The $A L D H 3 A 2$ gene is expressed in most mammalian tissues.

FALDH is a microsomal enzyme that oxidizes mediumand long-chain aliphatic aldehydes derived from metabolism of fatty alcohol, phytanic acid, ether glycerolipids and leukotriene B4 (Rizzo 2007). Fatty alcohols are oxidized by a fatty alcohol:NAD ${ }^{+}$oxidoreductase enzyme complex consisting of two protein components, fatty alcohol dehydrogenase and FALDH, which sequentially metabolize fatty alcohol to fatty aldehyde and fatty acid, respectively (Ichihara et al. 1986). As a consequence of FALDH deficiency, SLS patients have impaired hexadecanol oxidation (Rizzo et al. 1988) and accumulate long-chain fatty alcohols in cultured fibroblasts and plasma (Rizzo and Craft 2000). FALDH deficiency also leads to accumulation of leukotriene B4 (Willemsen et al. 2001b) and aldehydemodified phosphatidylethanolamine (James and Zoeller 1997). Altered membrane lipid composition in skin and brain is thought to be responsible for the symptoms in SLS (Rizzo 2007).

A number of mutations involving the $A L D H 3 A 2$ gene have been reported in SLS patients (De Laurenzi et al. 1996; Rizzo 2007; Rizzo and Carney 2005; Sillen et al. 1998; Willemsen et al. 2001a). Here we describe diseasecausing mutations in two Italian patients.

\section{Materials and methods}

Patients

All clinical investigations were approved by the Institutional Review Boards, according to the Helsinki Declaration, and performed after obtaining informed consent.
Electron microscopy

Electron microscopy samples were prefixed with $2 \%(\mathrm{w} / \mathrm{v})$ glutaraldehyde for $2 \mathrm{~h}$ at $4{ }^{\circ} \mathrm{C}$; complete fixation was achieved by incubation with $1 \%(\mathrm{w} / \mathrm{v})$ osmium tetroxide in $0.1 \mathrm{M}$ cacodylate buffer, $4.5 \%(\mathrm{w} / \mathrm{v})$ sucrose, for $1 \mathrm{~h}$ at $4^{\circ} \mathrm{C}$. Samples were dehydrated in ethanol and embedded in epoxy resin. Semithin sections $(1 \mu \mathrm{m})$ of embedded samples were cut using a microtome and stained with uranyl acetate $1 \%(\mathrm{w} / \mathrm{v})$.

Fibroblast culture

Cultured skin fibroblasts were grown from a skin biopsy of patient 2 using standard techniques.

RT-PCR and sequence analysis

RNA was isolated from cultured fibroblasts of patient 2 and a skin biopsy of patient 1 using the RNeasy Mini Kit (Qiagen, Crawley, UK). Randomly primed cDNA synthesis was performed using the ImProm-II Reverse Transcription System Kit (Promega). The FALDH coding region was amplified by PCR using primers: (+)ATTGTGGCTGT GGGTTGAGG and (-)AGAGGCACTAGGAGGTTGAA CAGG.

PCR amplification of the cDNA spanning exons 1 to 4 was performed using the following primers: (+)ATTGT GGCTGTGGGTTGAGG and (-)ACAATGTCCAGGT CACAATC.

\section{Genomic DNA analysis}

DNA was extracted and purified from blood samples using Wizard Genomic DNA Purification Kit (Promega). The ALDH3A2 gene was amplified by PCR using primers derived from genomic intronic sequences flanking the ALDH3A2 exons (Table 1). PCR was performing by adding $500 \mathrm{ng}$ of gDNA to a $50-\mu \mathrm{l}$ PCR reaction. The PCR product size and quality were checked on an agarose gel. DNA was purified from the agarose gel using the SV DNA Gel Purification Kit (Promega) and directly sequenced using the amplification primers and a 377 automatic sequencer (Applied Biosystems). ALDH3A2 haplotypes were determined as described (Rizzo et al. 1999).

FALDH enzyme activity

The FALDH enzyme activity in cultured fibroblasts was measured as previously described (Kelson et al. 1997). 
Table 1 Forward and reverse primer sequence, and size of PCR products, based on NT_030843 genomic contig $(01 / 02)$

\begin{tabular}{|c|c|c|c|c|}
\hline Exon no & Strand & Sequence & Name & Size (bp) \\
\hline \multirow[t]{2}{*}{1} & + & TGTGGCTGTGGGTTGACGGT & FALDHex1Forw & \multirow[t]{2}{*}{352} \\
\hline & - & AGCTCCAGTCCACAAGTGCG & FALDHex1Rev & \\
\hline \multirow[t]{2}{*}{$2-3$} & + & TGAATGGCAAACAGCTAGTC & FALDHex2-3Forw & \multirow[t]{2}{*}{2,057} \\
\hline & - & CCGAACATGTCCGAACA & FALDHex2-3Rev & \\
\hline \multirow[t]{2}{*}{$4-5$} & + & ATTTATTTGGCAGTGCAAGA & FALDHex4-5Forw & \multirow[t]{2}{*}{1,612} \\
\hline & - & GCTGCCTGCGAATAGTTAAT & FALDHex4-5Rev & \\
\hline \multirow[t]{2}{*}{6} & + & TGGCTGGATTTTGTACTTAC & FALDHex6Forw & \multirow[t]{2}{*}{349} \\
\hline & - & ACCAGAAAGCAGGATTTAGT & FALDHex6Rev & \\
\hline \multirow[t]{2}{*}{7} & + & GGGAGAGGGAAAGGCATGGA & FALDHex7Forw & \multirow[t]{2}{*}{430} \\
\hline & - & GCAACCTCGGAACACAACCT & FALDHex7Rev & \\
\hline \multirow[t]{2}{*}{8} & + & GGCCATGAGTGTTCCCTAAG & FALDHex8Forw & \multirow[t]{2}{*}{242} \\
\hline & - & TTTCAGCAGCCCATACAATC & FALDHex8Rev & \\
\hline \multirow[t]{2}{*}{9} & + & TTCCCGGTCGTTGTTAGA & FALDHex9Forw & \multirow[t]{2}{*}{465} \\
\hline & - & CAGTGACAAAAACGGGTAGC & FALDHex9Rev & \\
\hline \multirow[t]{2}{*}{$9^{\prime}$} & + & ACAGTATCCCAGCCTTAGTG & FALDHex9'Forw & \multirow[t]{2}{*}{230} \\
\hline & - & GCCСАССТТАСАТАTAGAAA & FALDHex $9^{\prime}$ Rev & \\
\hline \multirow[t]{2}{*}{10} & + & TTAAAGCAGCTGAGTAAACT & FALDHex10Forw & \multirow[t]{2}{*}{207} \\
\hline & - & GATGAATGGCAATGACT & FALDHex10Rev & \\
\hline
\end{tabular}

\section{Result and discussion}

\section{Case report}

One of the patients is a 12-year-old female. Ichthyosis became evident during the 1 st month of life. Due to spasticity in the legs, the patient first walked at 24 months of age only with support, on her tip-toes, and with adducted hips and flexed knees. She underwent surgical correction of leg contractures and started to walk independently with a spastic gait at 7 years of age. Since then, her motor disability has remained stable and currently, at 12 years of age, spasticity involves only the lower limbs. Pigmentary retinopathy was ruled out. Motor and sensory nerve conduction velocities were normal. Language development was normal, but the patient had moderate learning difficulties.

The other patient is a 5-year-old male. Ichthyosis present at birth and leg spasticity became evident in the subsequent few months, but the diplegia remained mild and surgical intervention has been avoided so far. Pigmentary retinopathy was ruled out, and he had a mild mental retardation.

In patient 2 a skin biopsy from the abdomen was processed for electron microscopy study. Semithin section examination showed orthokeratotic hyperkeratosis, thickening of the granular layer, acanthosis and papillomatosis (not shown). Electron microscopy revealed abnormal inclusions in the cytoplasm of granular and horny cells. In the granular layer several membrane-bound electron-lucent vacuoles were observed (Fig. 1a). These cytoplasmic inclusions often displayed peripherally located lamellar structures, suggesting that they may represent abnormal keratinosomes (also known as lamellar granules, Odland bodies, and membrane-coated granules). Numerous normalsized keratinosomes filled with parallel stacks of lamellae were also observed in the granular layer (not shown). The intercellular spaces of the lower stratum corneum appeared frequently widened and partly filled with a laminated or amorphous material (Fig. 1b). Within the horny layer cell cytoplasm, numerous vacuoles often containing lamellar structures were observed (Fig. 1b). Such abnormal lamellar inclusions in the granular and horny layer cells are a typical finding in SL syndrome and may be derived from keratinosomes, as already suggested by various authors (Bernardini et al. 2007; Ito et al. 1991; Rizzo 1993).

\section{Molecular analysis}

Mutation analysis of the $A L D H 3 A 2$ gene, revealed in the first patient a homozygous insertion mutation (c.769insA) in exon 5 (Fig. 1e), with parents heterozygous carriers (Fig. 1d). The c.769insA mutation consists of an adenine insertion into to a stretch of three adenines from 767 to $769 \mathrm{bp}$, resulting in a frame-shift and substitution of amino acids from Ile 257 to Ile 261 and a premature stop codon (TAA) at codon 262. The result is a severely truncated protein of only 261 amino acids. This mutation has not been previously reported in other SLS patients.

The second patient was also tested for FALDH enzyme activity in cultured skin fibroblasts, resulting severely

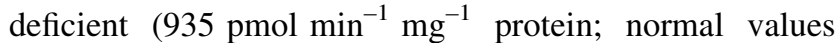
$6,750-20,570)$. Sequence analysis revealed the presence of 

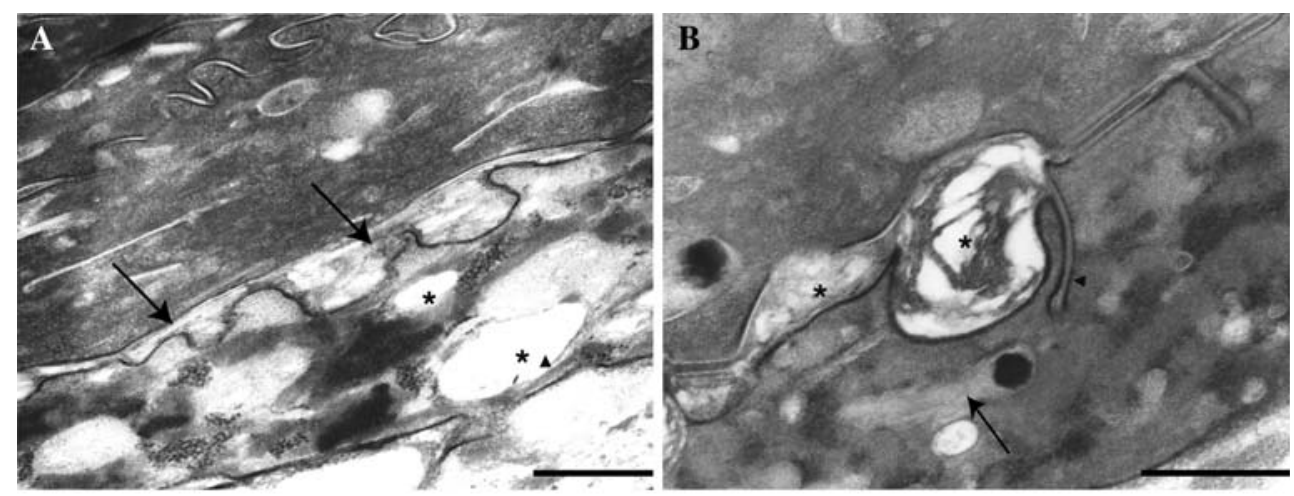

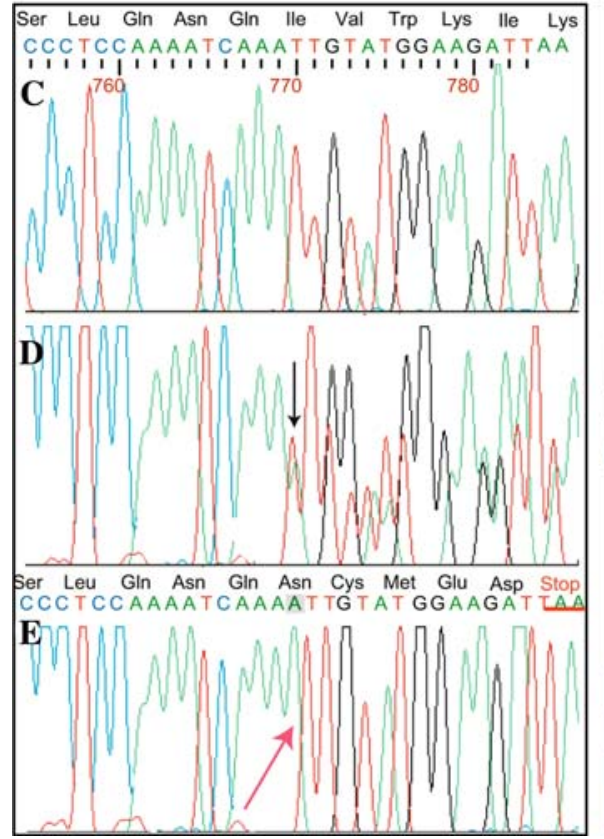

Fig. 1 Electron micrographs from involved skin. a Membrane-bound electron-lucent vacuoles (asterisks) in the granular layer: remnants of lamellar structures (arrowhead) are visible at the periphery of a vacuole. The intercellular space at the interface between the granular layer and the stratum corneum appear focally and irregularly enlarged, in particular at places of keratinosome fusion with the apical cell surface (arrows, bar $=500 \mathrm{~nm}$ ). b The intercellular spaces of the lower stratum corneum appear also focally widened and partly filled with a laminated or amorphous material (asterisk). Unusual plasma membrane invaginations containing parallel stacks of lamellae are also visible (arrowhead). Numerous vacuoles, which appear empty or filled with lamellar structures reminiscent of keratinosomes

two already described mutations: c.1094C $>$ T (Fig. 1f-g), leading to the substitution S365L (Shibaki et al. 2004; Sillen et al. 1998), and c.471 + 2T > G (Fig. 2a-b) (Rizzo et al. 1999). The heterozygous c. $471+2 \mathrm{~T}>\mathrm{G}$ splice site mutation found in patient 2 involves the donor splice-site (GT to GG) of exon 3. The mutation has been previously described (Rizzo et al. 1999), and causes skipping of exons 2 and 3 from the mRNA. The skipping results in the loss of 106 amino acids (52-157). Exon skipping caused by mutations affecting donor acceptor splice sites might not have full penetrance. Interestingly, cloning and sequencing

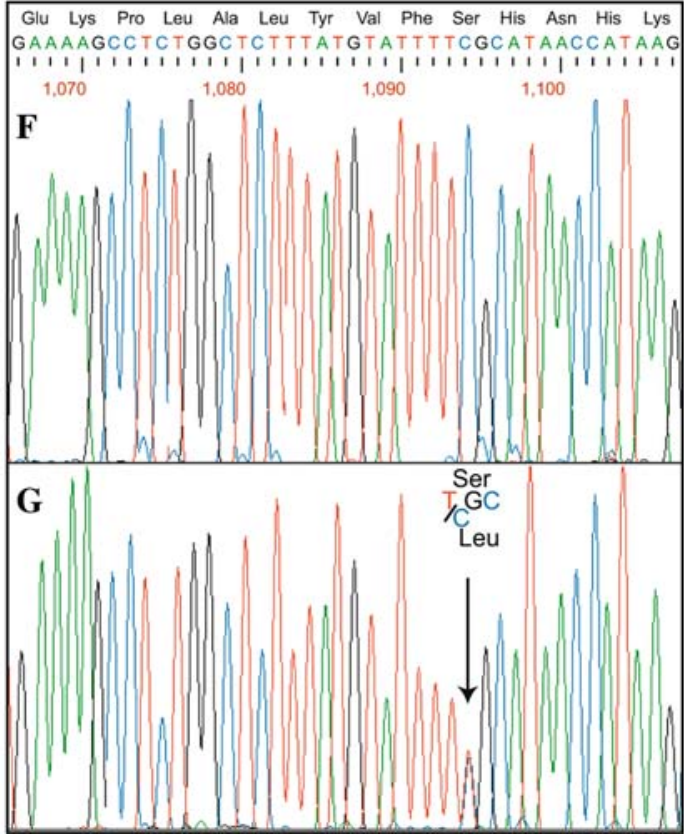

(arrow) and/or an amorphous material, are present within the cytoplasm of horny cells (bar $=200 \mathrm{~nm}$ ). Sequence analysis of the ALDH3A2 gene, exon 5 of patient 1 and exon 7 of patient 2 . c Wildtype sequence of exon 5. d Heterozygous mutation (present in both parents), showing the insertion of an adenine, the mutation is visible as a scrambling of the chromatogram (beginning from black arrow). e Homozygous insertion of an adenine into a stretch of three adenines (red arrow). This mutation leads to a frame-shift with a premature stop codon, TAA, underlined in red. f Wild-type sequence of exon 7. g Heterozygous mutation that results in $\mathrm{C}$ to $\mathrm{T}$ transversion (black arrow), determining the aminoacid substitution $\mathrm{S} 365 \mathrm{~L}$ in patient 2

of the full length cDNA (50 clones, Fig. 2c) only showed the presence of the mutated $\mathrm{S} 365 \mathrm{~L}$ allele (data not shown), thus demonstrating that the exon skipping is essentially complete.

The c.1094C $>\mathrm{T}$ mutation has been reported to be associated with two ALDH3A2 haplotypes (\#1 and \#2) (Rizzo and Carney 2005; Rizzo et al. 1999). We found that this patient was heterozygous for haplotypes \#1 and \#3; while the mother (c.471 + 2T > G) was heterozygous for haplotype 1 and 4 , and the father $(\mathrm{c} .1094 \mathrm{C}>\mathrm{T})$ was homozygous for haplotype \#3. The c.1094C > T mutation 
Fig. 2 Sequence analysis of the ALDH3A2 gene, exons 1-4, of patient 2. The entire CDS was amplified, and the two products obtained directly sequenced. a The chromatograms analysis of the smaller product shows the deletion generating the skipping of exons 2 and 3 , resulting in the loss of 106 amino acids. b The chromatogram of the genomic sequence shows the heterozygous c. $471+2 \mathrm{~T} \rightarrow \mathrm{G}$ transversion (black arrow). c The electrophoresis analysis showing the full length and a smaller transcript lacking $318 \mathrm{bp}$ due to the skipping of exons $2-3$. In this case, the PCR was performed with primers amplifying exon 1 to exon 4 of the patient's cDNA (see Materials and methods)
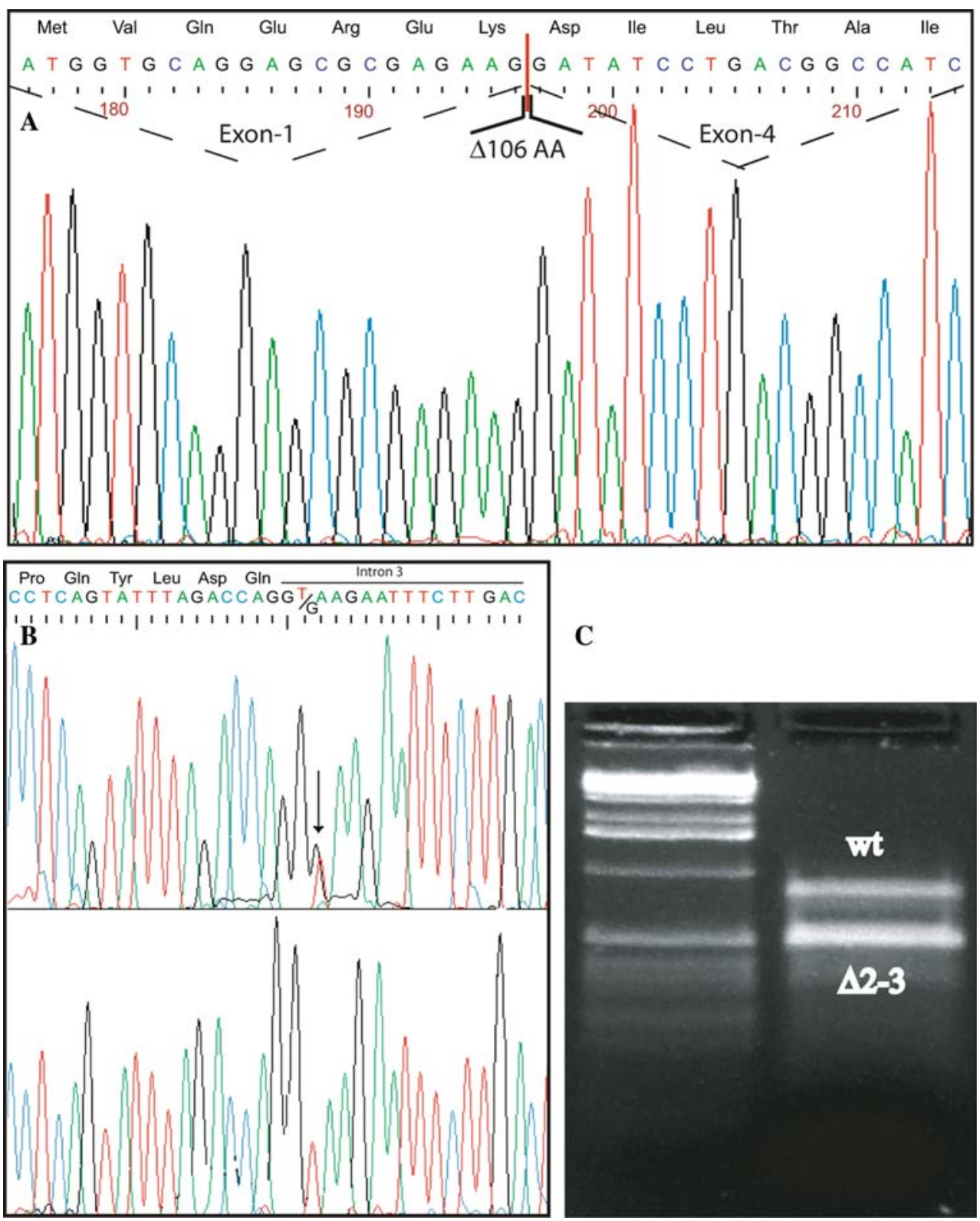

was therefore on $A L D H 3 A 2$ haploytpe \#3, indicating that this mutant allele represents a recurrent mutation on our Italian kindred.

We identified three mutations in the ALDH3A2 gene causing SLS in our Italian patients. The novel insertion mutation (c.767insA) in patient 1 gives rise to a severely truncated protein missing almost one-half of the amino acids, including a region essential for catalytic activity. The catalytic thiol of Cys241 is still present in the truncated protein, but other important residues are deleted, including: Glu333 recognized to be a general base in all class-3 aldehyde dehydrogenase enzymes (Hempel et al. 2001b); Glu268 important in activating the hydrolytic water after hydrite transfer (Hempel et al. 2001a); and Phe335 important for the positioning of the nicotinamide ring necessary for the hydrite transfer (Hempel et al. 2001a). In addition, this mutant protein lacks the hydrophobic carboxyl terminus, which is essential for anchoring it to the microsomal membrane (Masaki et al. 1994). This mutation should abolish enzyme catalytic activity, since a much less severe protein truncation (c.1297-1298delGA) results in $<1 \%$ of residual enzyme activity (Rizzo and Carney 2005). The c.767insA mutation occurs at a 3-bp adenine stretch separated by 2 -bp from an upstream four adenine sequence. This suggests that the mutation arose from a polymerase error at this site or unequal crossing over.

The c. 1094C $>$ T (S365L) mutation found in patient 2 has been reported in several SLS patients of German ancestry (Rizzo et al. 1999; Sillen et al. 1998). Expression studies have shown that the S365L mutant FALDH has only 3\% 
residual enzyme activity (Rizzo et al., 1999). The mutation occurs at a $\mathrm{CpG}$ dinucleotide and has been found to be associated with two ALDH3A2 haplotypes (\#1 and \#2), suggesting that it originated on at least two separate occasions. This mutation has also apparently arisen independently in an ancestor of our Italian patient, since it is associated with haplotype \#3. Our genetic studies lead us to speculate that the mutant c.1094C $>$ T allele in our Italian patient arose independently again in Italy. Interestingly this mutation has never been reported in non-Caucasians, such as the Japanese population (Sakai et al. 2006), indicating that this GT donor splice site is not prone to mutation in such a population, probably due to a different genetic backgrounds.

In summary, we report both novel and recurrent ALDH3A2 mutations in Italian SLS patients. These results provide new genetic findings on the molecular etiology of SLS and should be useful for DNA-based diagnosis of this disease.

Acknowledgments Contract grant sponsor: EU grants EPISTEM (LSHB-CT-019067), FIRB grants RBNE01KJHT_004,RBNE01NWCH_008; MIUR/PRIN 004064744_003; AIRC rif. 1338; ISS n. 530/F-A19. The work was also supported by Grant Telethon GGPO4110 to Gerry Melino, and ISS Programma Italia-USA "Malattie Rare" N.526D/5.

\section{References}

Bernardini ML, Cangiotti AM, Zamponi N, Porfiri L, Cinti S, Offidani A (2007) Diagnosing Sjogren-Larsson syndrome in a 7-year-old Moroccan boy. J Cutan Pathol 34:270-275

Chang C, Yoshida A (1997) Human fatty aldehyde dehydrogenase gene (ALDH10): organization and tissue-dependent expression. Genomics 40:80-85

De Laurenzi V, Rogers GR, Hamrock DJ, Marekov LN, Steinert PM, Compton JG, Markova N, Rizzo WB (1996) Sjogren-Larsson syndrome is caused by mutations in the fatty aldehyde dehydrogenase gene. Nat Genet 12:52-57

Hempel J, Kuo I, Perozich J, Wang BC, Lindahl R, Nicholas H (2001a) Aldehyde dehydrogenase. Maintaining critical active site geometry at motif 8 in the class 3 enzyme. Eur J Biochem 268:722-726

Hempel J, Lindahl R, Perozich J, Wang B, Kuo I, Nicholas H (2001b) Beyond the catalytic core of ALDH: a web of important residues begins to emerge. Chem Biol Interact 130-132:39-46

Ichihara K, Kusunose E, Noda Y, Kusunose M (1986) Some properties of the fatty alcohol oxidation system and reconstitution of microsomal oxidation activity in intestinal mucosa. Biochim Biophys Acta 878:412-418

Ito M, Oguro K, Sato Y (1991) Ultrastructural study of the skin in Sjogren-Larsson syndrome. Arch Dermatol Res 283:141-148

Jagell S, Polland W, Sandgren O (1980) Specific changes in the fundus typical for the Sjogren-Larsson syndrome. An ophthalmological study of 35 patients. Acta Ophthalmol (Copenh) 58:321-330

Jagell S, Gustavson KH, Holmgren G (1981) Sjogren-Larsson syndrome in Sweden. A clinical, genetic and epidemiological study. Clin Genet 19:233-256

James PF, Zoeller RA (1997) Isolation of animal cell mutants defective in long-chain fatty aldehyde dehydrogenase.
Sensitivity to fatty aldehydes and Schiff's base modification of phospholipids: implications for Sjogren-Larsson syndrome. J Biol Chem 272:23532-23539

Kelson TL, Secor McVoy JR, Rizzo WB (1997) Human liver fatty aldehyde dehydrogenase: microsomal localization, purification, and biochemical characterization. Biochim Biophys Acta 1335:99-110

Masaki R, Yamamoto A, Tashiro Y (1994) Microsomal aldehyde dehydrogenase is localized to the endoplasmic reticulum via its carboxyl-terminal 35 amino acids. J Cell Biol 126:1407-1420

Pigg M, Jagell S, Sillen A, Weissenbach J, Gustavson KH, Wadelius C (1994) The Sjogren-Larsson syndrome gene is close to D17S805 as determined by linkage analysis and allelic association. Nat Genet 8:361-364

Rizzo WB (1993) Sjogren-Larsson syndrome. Semin Dermatol $12: 210-218$

Rizzo WB (2001) Sjogren-Larsson syndrome: fatty aldehyde dehydrogenase deficiency. McGraw-Hill, New York, pp 2239-2258

Rizzo WB (2007) Sjogren-Larsson syndrome: molecular genetics and biochemical pathogenesis of fatty aldehyde dehydrogenase deficiency. Mol Genet Metab 90:1-9

Rizzo WB, Carney G (2005) Sjogren-Larsson syndrome: diversity of mutations and polymorphisms in the fatty aldehyde dehydrogenase gene (ALDH3A2). Hum Mutat 26:1-10

Rizzo WB, Craft DA (2000) Sjogren-Larsson syndrome: accumulation of free fatty alcohols in cultured fibroblasts and plasma. $\mathrm{J}$ Lipid Res 41:1077-1081

Rizzo WB, Dammann AL, Craft DA (1988) Sjogren-Larsson syndrome. Impaired fatty alcohol oxidation in cultured fibroblasts due to deficient fatty alcohol: nicotinamide adenine dinucleotide oxidoreductase activity. J Clin Invest 81:738-744

Rizzo WB, Carney G, Lin Z (1999) The molecular basis of SjogrenLarsson syndrome: mutation analysis of the fatty aldehyde dehydrogenase gene. Am J Hum Genet 65:1547-1560

Rogers GR, Rizzo WB, Zlotogorski A, Hashem N, Lee M, Compton JG, Bale SJ (1995) Genetic homogeneity in Sjogren-Larsson syndrome: linkage to chromosome $17 \mathrm{p}$ in families of different non-Swedish ethnic origins. Am J Hum Genet 57:1123-1129

Rogers GR, Markova NG, De Laurenzi V, Rizzo WB, Compton JG (1997) Genomic organization and expression of the human fatty aldehyde dehydrogenase gene (FALDH). Genomics 39:127-135

Sakai K, Akiyama M, Watanabe T, Sanayama K, Sugita K, Takahashi M, Suehiro K, Yorifuji K, Shibaki A, Shimizu H (2006) Novel ALDH3A2 Heterozygous mutations in a Japanese family with Sjogren-Larsson syndrome. J Invest Dermatol 126:2545-2547

Shibaki A, Akiyama M, Shimizu H (2004) Novel ALDH3A2 heterozygous mutations are associated with defective lamellar granule formation in a Japanese family of Sjogren-Larsson syndrome. J Invest Dermatol 123:1197-1199

Sillen A, Anton-Lamprecht I, Braun-Quentin C, Kraus CS, Sayli BS, Ayuso C, Jagell S, Kuster W, Wadelius C (1998) Spectrum of mutations and sequence variants in the FALDH gene in patients with Sjogren-Larsson syndrome. Hum Mutat 12:377-384

Sjogren T, Larsson T (1957) Oligophrenia in combination with congenital ichthyosis and spastic disorders; a clinical and genetic study. Acta Psychiatr Neurol Scand Suppl 113:1-112

Willemsen MA, L IJ, Steijlen PM, Rotteveel JJ, de Jong JG, van Domburg PH, Mayatepek E, Gabreels FJ, Wanders RJ (2001a) Clinical, biochemical and molecular genetic characteristics of 19 patients with the Sjogren-Larsson syndrome. Brain 124:14261437

Willemsen MA, Rotteveel JJ, de Jong JG, Wanders RJ, L IJ, Hoffmann GF, Mayatepek E (2001b) Defective metabolism of leukotriene B4 in the Sjogren-Larsson syndrome. J Neurol Sci 183:61-67 